\title{
Know Your Neighbour: Packet Loss Correlation in IEEE 802.11b/g Multicast
}

\author{
Janus Heide \\ Department of Electronic \\ Systems \\ Aalborg University, Denmark \\ speje@es.aau.dk
}

\author{
Morten V. Pedersen \\ Department of Electronic \\ Systems \\ Aalborg University, Denmark \\ mvpe@es.aau.dk
}

\author{
Frank H.P. Fitzek \\ Department of Electronic \\ Systems \\ Aalborg University, Denmark \\ ff@es.aau.dk
}

\author{
Tatiana K. Madsen \\ Department of Electronic \\ Systems \\ Aalborg University, Denmark \\ tatiana@es.aau.dk
}

Torben Larsen

Department of Electronic Systems

Aalborg University, Denmark tl@es.aau.dk

\section{Keywords}

\begin{abstract}
In this paper we present a number of wireless measurements conducted on mobile phones using WLAN IEEE802.11b/g in a multicast scenario. When designing efficient error recovery mechanisms for reliable multicast protocols, it is necessary to have good a understanding how channel errors occur and preferably to have a network model that describes these errors well. The measurement results in this work are not sufficient to create an accurate error model, nevertheless they provide two important results. First, the assumption of independent package loss, commonly assumed in simulations and analytical work, does not hold. In all measurements conducted the packet loss, observed at different devices, indicated some level of correlation. Therefore error models used for simulations and analytical evaluation should take this finding into consideration. Secondly, the packet loss probability observed at mobile devices of the same brand and model, exhibited large differences. A uniform packet error probability will therefore not be a satisfactory error model, even when considering homogeneous networks.
\end{abstract}

\section{Categories and Subject Descriptors}

C.2.1 [Computer Systems Organization]: ComputerCommunication Networks-Network Architecture and Design; C.4 [Computer Systems Organization]: Performance of Systems

\section{General Terms}

Measurement, Performance, Reliability

Mobimedia 2008 July 7-9, 2008, Oulu, Finland. Copyright 2008 ICST ISBN 978-963-9799-25-7/08/07 DOI 10.4108/ICST.MOBIMEDIA2008. 4020
Multicast, Packet loss, Mobile phones, 802.11b/g

\section{INTRODUCTION}

The amount of group oriented applications such as multimedia streaming, distributed gaming, etc. has increased in resent years. In such services, distributing data using multicast provides an efficient utilization of the available bandwidth [1], and is considered a key technology in future wireless networks [2]. As a consequence a large research effort has been put into designing multicast protocols for routing and data distribution, especially in Wireless Mesh Networks (WMNs) and Mobile Ad-hoc NETworks (MANETs). One of the significant technical challenges when designing efficient multicast protocols, is how to provide high data rates and reliable end-to-end communication. While at the same time ensuring that the desired Quality of Service (QoS) for the specific application is maintained. Designing such protocols requires a good understanding of several factors such as application requirements, network topologies and packet loss characteristics [3]. When designing error recovery strategies one commonly made assumption is that the loss of packets is uncorrelated within a group of receiving nodes $[4,5,6]$. If this assumption is not accurate it can have a substantial impact on the performance of the protocol [7].

The consequence of correlated packet losses can be illustrated by considering a simple wireless network as shown in Figure 1(a). In this scenario one sending node denoted $s$, multicasts data to six receivers $\{n 1, \ldots, n 6\}$. The punctured circles represent which nodes are within communication range of each other. First let us assume that the packets transmitted from $s$ are lost with independent probabilities $p_{1}$ and $p_{2}$ at node $n 1$ and $n 2$ respectively. If the two individual loss probabilities are reasonably low, we have a very small joint probability that both receivers loose the same packet given by $p_{1} p_{2}$. Thereby if a packet is lost at $n 1$, one simple and efficient error recovery scheme will be to let $n 1$ wait until $n 2$ transmits the packet to node $n 3$ and $n 4$. This type of passive error recovery strategies will work most of the time, if packet losses are uncorrelated. Alternatively consider if node $n 1$ and $n 2$ always looses the same packets as illustrated in Figure 1(b), in this case it would not make 
sense for $n 1$ to wait until $n 2$ transmits the packet. In such a highly correlated setup the best strategy would be to let $s$ retransmit the packet as soon as possible. Hence knowing to what degree the packet losses are correlated will allow us to choose the most efficient strategy.

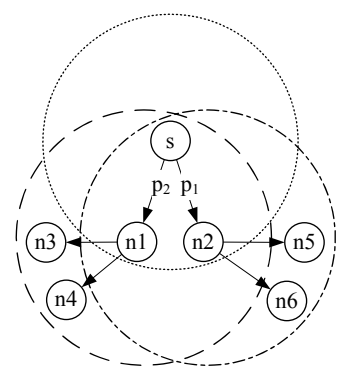

(a) Uncorrelated packet losses

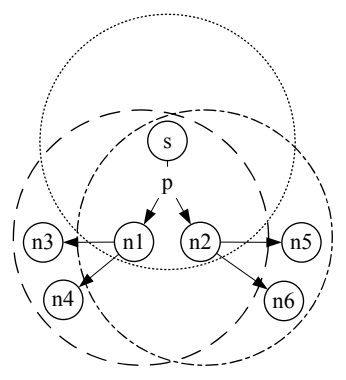

(b) Correlated packet losses
Figure 1: Two examples illustrating how packet loss correlation at different receivers can affect the error recovery mechanism used.

(a) the packet losses are uncorrelated. When error probabilities $p_{1}$ and $p_{2}$ are low, one of the receivers $\{n 1, n 2\}$ within the senders $s$ transmit range will likely receive the packet. Hence if $n 1$ looses a packet it can wait and receive the packet when node $n 2$ transmits the packet to $n 5$ and $n 6$.

(b) the errors are highly correlated. If a packet is lost both devices will lose it, in this case a good error recovery strategy is to await a retransmission from the sender $s$.

Despite the packet loss correlations impact on the performance of error recovery aspects in multicast based protocols, so far little attention has been given to investigate the packet loss correlation between neighboring devices. In [7] loss correlation has been determined between a small number of Wireless Local Area Network (WLAN) equipped laptops. The results are used to compare video streaming using leader based Automatic Repeat-reQuest (ARQ) schemes and legacy multicast. Unfortunately the paper does not describe how the correlation was calculated making it difficult to verify the results. In [8] several error control mechanisms are evaluated using packet loss profiles built from real traces. These traces have been obtained without considering losses that occur before the multicast traffic is in the air, e.g. due to overflow in the Access Point (AP) send buffer. Also no classification of the level of interference is performed. In [9] measurements using WLAN show that packet losses at several devices can exhibit a certain degree of correlation. To account for the observed correlation and break with traditional error models (Gilbert-Elliot and Bernoulli) Lacan et al. proposes a new approach for simulating packet losses, which more accurately describes the observed correlation. Unfortunately the traces gathered do not take into account losses occurring before data is transmitted from the AP.

Another parameter affecting packet loss characteristics are the hardware variations which causes differences in link quality between nodes. In [10] Fuxjäger et al. observes that the frame-error-rates of identical network interfaces can vary sig- nificantly. The importance of handling these variations is illustrated in the simple network shown in Figure 2. In this multi-hop setup one sender $s$, conveys a stream of packets to the nodes $n 3$ and $n 4$. Assume that node $n 1$ has better performance characteristics than $n 2$. In this case when $s$ transmits a packet, node $n 1$ should be chosen as the relay. If both relays are exhibiting similar performance characteristics either can be chosen as the relay. The measurement campaign will give an indication of whether packet error rates vary to such an extent, between identical devices, that future protocols should take this into account.

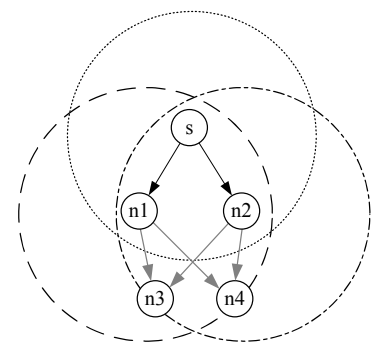

Figure 2: Multi-hop network where different relays exist. An efficient multicast protocol should choose the best relay when transmitting packets from the sender $s$ to the receivers $n 3$ and $n 4$.

In general WLAN measurement campaigns found in current research are carried out using laptops or stationary computers. In this paper the measurements are carried out using our 802.11 capable mobile phone testbed. Therefore in addition to the loss characteristics, this will give an insight into the performance in terms of delay and throughput obtainable on state-of-the-art mobile phones. The results can be the basis for work on reliable multicast data distribution in mobile networks, but could also be interesting for researchers in related fields as it will provide real-life data, which could be used for performance analysis of wireless networks using mobile phones.

In this paper we will focus on answering the following questions:

- What is the spatial correlation of packet losses between nodes in a single hop network?

- Will adjusting the transmit power or preventing Line Of Sight (LOS) between sender and receiver affect the loss correlation observed?

- Can we measure significant variability in the application layer packet error probabilities from phone to phone (when using identical phones)?

The remainder of the paper is organized as follows. In Section 2 the testbed setup is presented and the tools developed are described. Section 3 describe the post-processing steps and how the correlation was quantified. The traces obtained in the measurement campaign are presented in Section 4. The final conclusions are drawn in Section 5.

\section{MEASUREMENT SETUP}

The measurement campaign was carried out at Aalborg University, Denmark, in a public space. The setup consisted of one laptop connected to a wireless access point, 
nine mobile devices (Nokia N95), and a laptop functioning as a network sniffer. The latter was used to characterize the wireless environment in which the measurements were conducted. This was necessary as no control over the interfering traffic from other WLANs was possible. The measurement area and setup is shown in Figure 3.

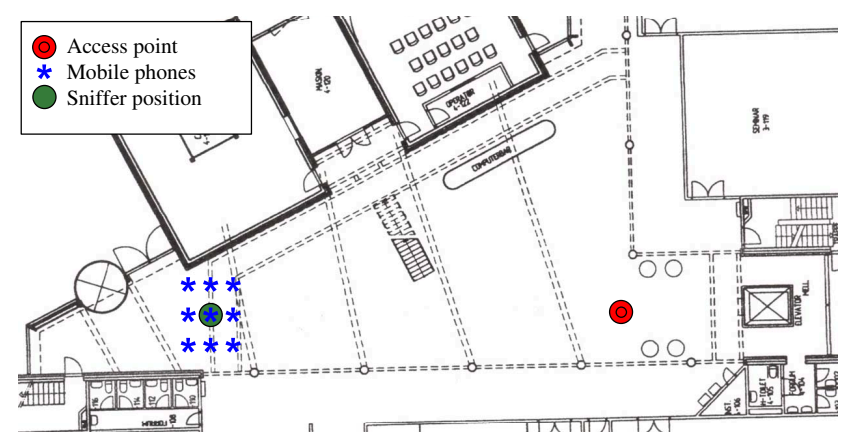

Figure 3: Position of mobile devices and AP used in the measurements. The distance from the AP to the receiving devices is $25 \mathrm{~m}$.

Figure 3 shows the nine mobile phones places in a $3 \times 3$ grid. Two different grid sizes were used with inter-node distance of $30 \mathrm{~cm}$ and $1 \mathrm{~m}$ respectively. The network sniffer was placed below the receiving phones. The sending AP was located $25 \mathrm{~m}$ from the centre of the grid. The following equipment was used in the measurement.

- Laptop: An IBM T40 running Windows XP SP2, was used as packet generator. The laptop was connected to the AP with an Ethernet 10/100 TBase connection.

- Access Point: A Cisco Arionet 1100 series AP was used as it provided the necessary control over transmission power and made it easy to monitor sent packets. The monitor capability was important as it allowed us to verify that no packets were lost between the sending laptop and the AP e.g. in the APs transmit buffer.

- Laptop: One IBM R50 running Ubuntu 7.10 k2.6.22.14, was used as packet sniffer. To perform the sniffing Wireshark [11] was used. The laptops internal WLAN card was set in promiscuous mode, allowing Wireshark to capture interfering network traffic.

- Mobile Phones: As receiving terminals we used nine Nokia N95s, running Symbian OS 9.2 (firmware version 21.0.016).

The measurements were carried out using mobile phones as the receiving devices. Therefore we developed a new tool, named NetMon, to carry out the measurements and automate the testing procedure. NetMon consists of a client and server part. The server runs on the sending laptop and can be configured to perform different tests. In these measurements it was configured to generate a traceable packet stream, consisting of a unique test and message identifier. This packet stream was transmited, via the AP, to the nine mobile devices running the NetMon client. The mobile devices gathered the received data and stored this in a trace file. After completing a test the results were retrieved from the mobile devices by the NetMon server. The NetMon server also controlled the sniffer thereby fully automating the test procedure. After completing a measurement the trace files and results from the sniffer laptop was processed using Python and Matlab scripts. The post processing is described in the following section.

\section{DATA PROCESSING}

In this section the data processing conducted on the measurement tracefiles is described. In order to quantify the correlation of losses experienced at different receivers the Kullback-Leibler (KL) distance is introduced [12]. This was used to compare the erasure-processes taken from the measurement data and a entirely uncorrelated erasure-process.

For each packet transmitted, between zero and all nodes (here nine) can receive the packet. In order to determine how many nodes lost each packet, denoted $\bar{e}$ we sum all error-vectors, $\bar{e}_{j}$, where $e(i)_{j} \in\{0,1\}$ and $e(i)_{j}=1$ indicates an erasure for packet $i$ in node $j$.

$$
\bar{e}=\sum_{j=1}^{n} \bar{e}_{j}
$$

where:

$\bar{e}_{j}$ is the error-vector for node $j$.

$n$ is the number of nodes.

\subsection{Data Rate \& Packet Error Probability}

The data rate $D_{j}$ was found for each node by dividing the amount of received data with the transmission time. The cluster data rate is denoted $\bar{D}$. The Packet Error Probability (PEP) for each node $P_{e r r, j}$ was calculated by summing its error-vector and dividing with the number of transmitted packets from the AP. The cluster PEP is denoted $\bar{P}_{e r r}$.

\subsection{Independent Erasure Processes}

Given the set $R_{n}=\left\{r_{1}, r_{2}, \ldots, r_{n}\right\}$ of $n$ independent receivers with identical PEP, $P_{e r r}(\bar{r})=P_{e r r}\left(r_{1}\right)=P_{e r r}\left(r_{2}\right)=$ $\ldots=P_{\text {err }}\left(r_{n}\right)$. The PEP for $m$ receivers $\in R_{n}$ is given by Equation (2).

$$
P_{\text {err }}(m)=P_{e r r}(\bar{r})^{m}
$$

\subsection{Kullback-Leibler distance}

To calculate the KL distance we first determine the number of nodes which lost packet $i, \bar{e}(i)$, for $i=\{1,2, \ldots, k\}$, where $k$ is the total number of transmitted packets. From this the Probability Mass Function (pmf) of the cluster is constructed. This can be compared to the theoretical pmf constructed from the assumption that all $n$ nodes are uncorrelated and from the mean PEP experienced in the measurements. The KL distance between the theoretical and measured pmf is defined by Equation (3).

$$
D(p \| q)=\sum_{x \in X} p(x) \log \frac{p(x)}{q(x)}
$$

The KL distance expresses the distance between two pmfs however this measure is not symmetric [13]. In order to avoid any confusion, whenever we calculate the KL distance $p$ is always the true pmf constructed from Equation (2) and $q$ is always the target pmf of the measured packet errors. 


\section{RESULTS}

In this section we present the results of the conducted experiments. From these we determine if the loss of packets at each node is more correlated than traditionally assumed. To determine this we will use the approach and equations defined in Section 3. The testbed was created as described in Section 2. The AP was configured to use a fixed data rate of $11 \mathrm{Mbit} / \mathrm{s}$ and a transmit power of 1, 5, 20, 30 and $50 \mathrm{~mW}$ respectively. For each of these settings the AP transmitted 10000 packets of $1400 \mathrm{~B}$ and each test was performed ten times. This was done with Line Of Sight (LOS) between the $\mathrm{AP}$ and the cluster and with Non Line Of Sight (NLOS).

The data rate and packet loss in the grid has been calculated for all measurements and is listed in Table 1. From these results it can be seen that the LOS measurements in general have lower data rate (column $D$ ) and higher loss probability (column $L$ ) compared to the NLOS measurements. This appears counter intuitive as we would expect that the transmission conditions would deteriorate when LOS was prevented. However if we consider the measured interference (column $I$ ) we see that during the LOS measurements, the magnitude of interfering traffic captured by the sniffer is generally higher compared to the NLOS measurements. This indicates that the level of interfering traffic has a higher impact on the loss rate, compared to LOS or NLOS between the sender and receivers. In addition there seems to be no apparent trend in the packet losses with respect to transmission power (column $P$ ).

Note the packet loss for the measurement where packets are sent continuously ( $0 \mathrm{~ms}$ spacing, column $\delta S$ ) from the server. In this measurement the nodes losses approximately three times the number of packets compared to the measurement where the server pauses (4 ms spacing) between each packet. This effect is not surprising as an unreliable protocol allows packets to be dropped without any notification. This stresses the necessity to consider this potentially dominating source of errors, when measurements with UDP packets is carried out.

\begin{tabular}{|c|c|c|c|c|c|c|c|}
\hline \multirow[b]{2}{*}{$\begin{array}{c}\mathrm{P} \\
{[\mathrm{mW}]}\end{array}$} & \multirow[b]{2}{*}{$\begin{array}{c}\Delta \mathrm{S} \\
{[\mathrm{ms}]}\end{array}$} & \multicolumn{3}{|c|}{ Line of Sight } & \multicolumn{3}{|c|}{ Non Line of Sight } \\
\hline & & $\begin{array}{c}\mathrm{D} \\
{[\mathrm{Mbps}]}\end{array}$ & $\begin{array}{c}\mathrm{L} \\
{[\%]}\end{array}$ & $\begin{array}{c}\mathrm{I} \\
{[\%]}\end{array}$ & $\begin{array}{c}\mathrm{D} \\
{[\mathrm{Mbps}]}\end{array}$ & $\begin{array}{c}\mathrm{L} \\
{[\%]}\end{array}$ & $\begin{array}{c}\mathrm{I} \\
{[\%]}\end{array}$ \\
\hline 1 & 4 & 1.82 & 33.0 & 12.29 & 1.82 & 33.0 & 7.92 \\
\hline 5 & 4 & 1.63 & 40.0 & 11.16 & 1.90 & 30.2 & 5.55 \\
\hline 20 & 4 & 1.80 & 33.7 & 18.24 & 1.95 & 28.1 & 10.67 \\
\hline 30 & 4 & 1.71 & 36.9 & 17.07 & 2.08 & 23.4 & 12.90 \\
\hline 50 & 4 & 1.51 & 44.4 & 13.00 & 1.87 & 31.1 & 8.39 \\
\hline 50 & 0 & 2.29 & 88.9 & - & 2.29 & 89.0 & - \\
\hline
\end{tabular}

Table 1: Data rates and packet losses for the receivers in the cluster at different test conditions

From the measurement data we can estimate a pmf of the number of errors that occur. From the experienced PEP we can construct the theoretical pmfs from Equation (2) under the assumption that packet losses are uncorrelated. We then compare these pmfs in Figure 4. In order to quantify the difference between the theoretical and measured packet loss, the Kullback-Leibler distance has been calculated with Equation (3), see Table 2.

All plots in Figure 4 and its corresponding Kullback-Leibler distance in Table 2, indicate that the occurrence of higher order erasures are greater than predicted under the assump-

\begin{tabular}{|c|c|c|}
\hline \multirow{2}{*}{$\begin{array}{c}\text { Power } \\
{[\mathrm{mW}]}\end{array}$} & \begin{tabular}{c} 
Line of Sight \\
\cline { 2 - 3 }
\end{tabular} & $\begin{array}{c}\text { Non Line of Sight } \\
{[-]}\end{array}$ \\
\hline 1 & 0.0183 & $\begin{array}{c}\text { KL distance } \\
{[-]}\end{array}$ \\
\hline 5 & 0.0014 & 0.0571 \\
\hline 20 & 0.0072 & 0.0075 \\
\hline 30 & 0.0188 & 0.0040 \\
\hline 50 & 0.0091 & 0.0131 \\
\hline
\end{tabular}

Table 2: The KL distance at different test conditions

tion of independent packet losses. Hence packet losses are not uncorrelated in the performed test, neither in LOS scenario, nor in the NLOS scenario. However from Figure 4 it is evident that uncorrelated packet losses are a good approximation when the number of devices are small, $\in\{2,3\}$, but becomes increasingly inaccurate as the number of devices grows.

To determine whether the PEPs between identical devices variates significantly. We performed two control tests where the only difference was the position of the devices in the grid, see Figure 5, 6 and Figure 7, 8. For all nine nodes in the cluster, the number of received packets is plotted for ten consecutive tests.

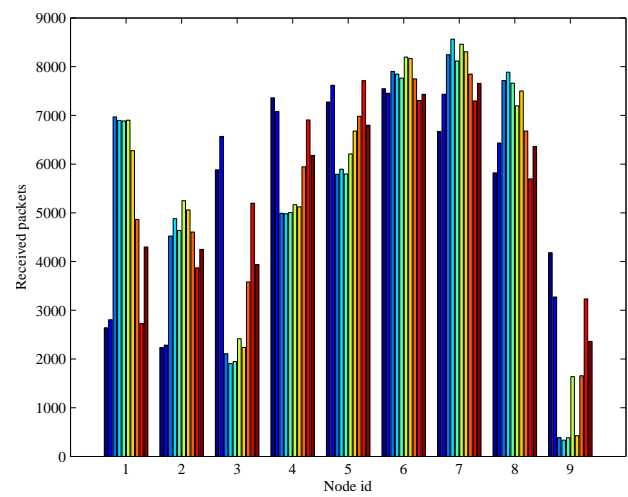

\begin{tabular}{|l|l|l|}
\hline 8 & 6 & 7 \\
\hline 1 & 4 & 5 \\
\hline 2 & 3 & 9 \\
\hline
\end{tabular}

Figure 6: Node locations 1

Figure 5: Packets received for each node for ten tests
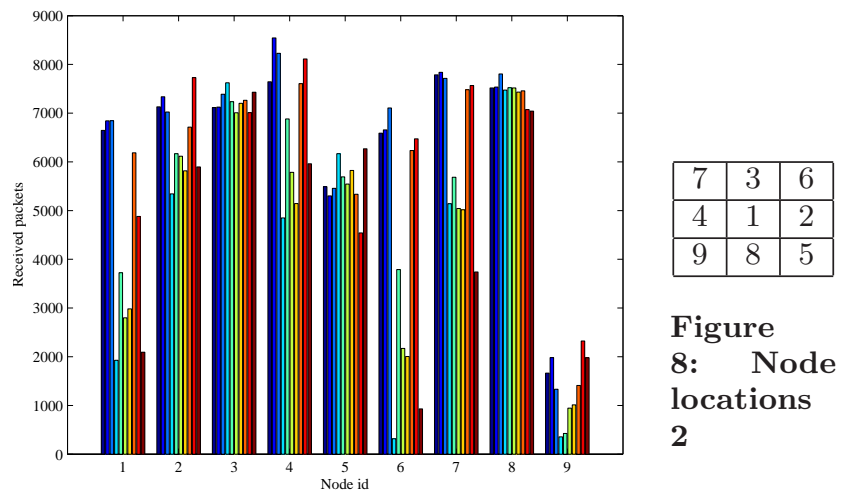

Figure

8: $\quad$ Node locations 2

Figure 7: Packets received for each node for ten tests

The PEP for the two measurements is 0.401 and 0.375 respectively. From Figure 5 and 7 we can see that de- 


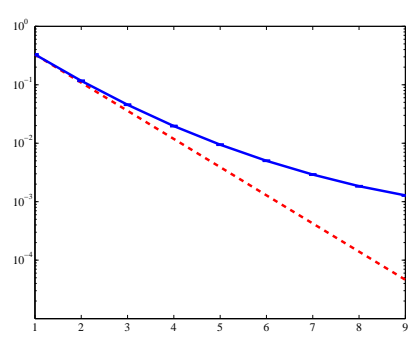

(a) $1 \mathrm{~mW}$, LOS

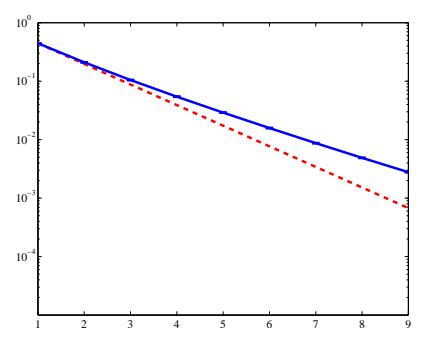

(e) $50 \mathrm{~mW}, \mathrm{LOS}$

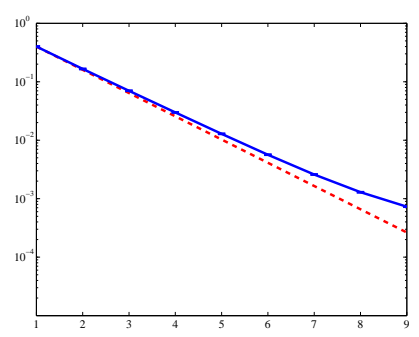

(b) $5 \mathrm{~mW}$, LOS

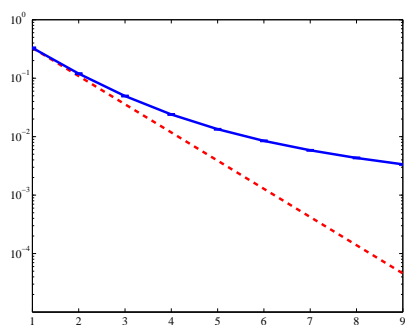

(f) $1 \mathrm{~mW}$, NLOS

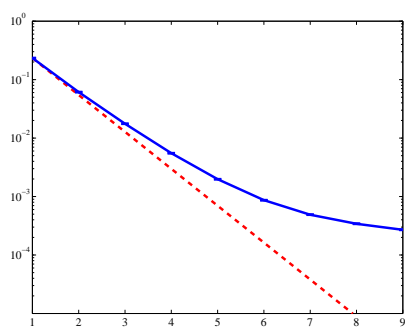

(i) $30 \mathrm{~mW}, \mathrm{NLOS}$

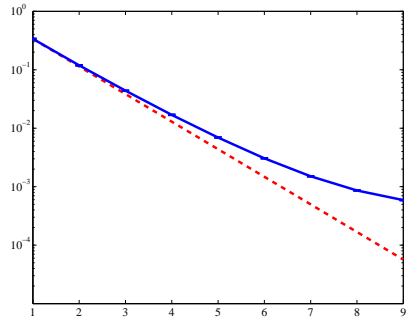

(c) $20 \mathrm{~mW}$, LOS

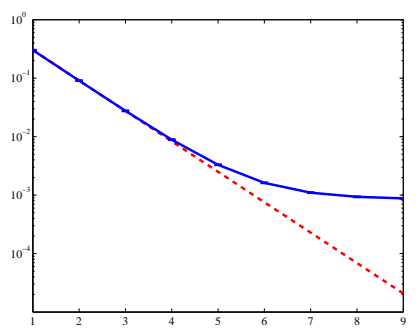

(g) $5 \mathrm{~mW}$, NLOS

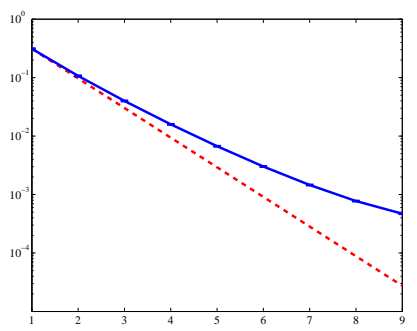

(j) $50 \mathrm{~mW}, \mathrm{NLOS}$

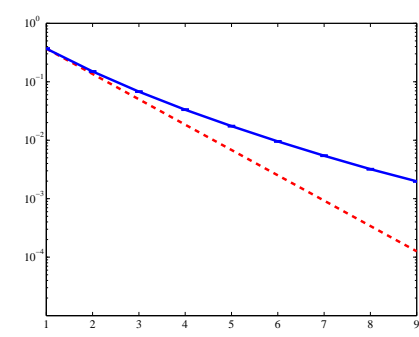

(d) $30 \mathrm{~mW}$, LOS

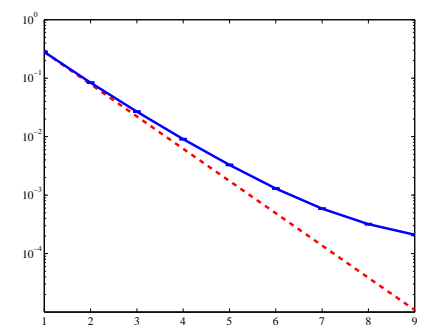

(h) $20 \mathrm{~mW}$, NLOS

Figure 4: The theoretical and measured probability of different number of erasures for a packet transmitted to the cluster. The punctured line is the theoretical probabilities under the assumption of independent erasure processes, the solid line is the estimated probabilities found from the collected data.

vice number 9 performs significantly below average in both tests. The performance of the rest of the devices variate to different extents. From this it is evident that some devices have considerably worse characteristics than others. If we observe the performance of the different positions in the two tests, no single position gives bad results in both tests. Hence the location does not significantly influence the PEP of the devices in this measurement. For a strong conclusion to be made more measurements should be conducted, however these measurements indicate that the quality of the devices can variate considerably. This variation can obviously greatly influence conducted measurements and precaution should therefore be taken to minimize its influence.

To determine whether the inter-node distance in the cluster influence correlation, we conducted a control test where the distance of the nodes was increased to $1 \mathrm{~m}$. The $\mathrm{KL}$ distance in this measurement was close to the KL distance of the test with $30 \mathrm{~cm}$ inter-node distance. Hence the correlation did not appear to be influenced by a distance change in this order of magnitude in this particular test setup. In general the correlation is expected to be influenced if we continue to increase the inter-node distance.

\section{CONCLUSION \& FUTURE WORK}

In this paper we introduced our IEEE 802.11 phone testbed and NetMon measurement tools. These were used to carry out a preliminary study of the packet loss correlation in a grid of mobile phones using a multicast transmission scheme. The results show that packet losses always exhibit a certain level of correlation, and that the correlation does not seem to be influenced by transmission power or line of sight. Further it is found that identical devices performs quite differently in terms of PEP.

In our future work, we will focus on exploring other factors which may also influence the correlation. In addition we plan to carry out an extensive follow-up measurement campaign, in order improve the statistically significance of the 
gathered results. We further plan to publish the packet trace files online, to allow others to access the traces obtained in different scenarios.

\section{ACKNOWLEDGEMENT}

We would like to thank Nokia for providing technical support as well as mobile phones to carry out the measurement campaign. Special thanks to Mika Kuulusa, Gerard Bosch, Harri Pennanen, Nina Tammelin, and Per Moeller from Nokia. This work was partially financed by the X3MP project granted by Danish Ministry of Science, Technology and Innovation.

\section{REFERENCES}

[1] J. Kuri and S. K. Kasera, "Reliable multicast in multi-access wireless LANs," in INFOCOM (2), pp. 760-767, 1999 .

[2] K. Bür and C. Ersoy, "Ad hoc quality of service multicast routing," Elsevier Science Computer Communications, vol. 29, no. 1, pp. 136-148, December 2005.

[3] H.-S. W. So, K. Fall, and J. WalrandIn-Stat, "Packet loss behavior in a wireless broadcast sensor network," tech. rep., University of California, Berkeley, 2003.

[4] S. Pingali, D. Towsley, and J. F. Kurose, "A comparison of sender-initiated and receiver-initiated reliable multicast protocols," in SIGMETRICS '94: Proceedings of the 1994 ACM SIGMETRICS conference on Measurement and modeling of computer systems, (New York, USA), pp. 221-230, ACM, 1994.

[5] J.-S. Park, M. Gerla, D. S. Lun, Y. Yi, and M. Medard, "Codecast: a network-coding-based ad hoc multicast protocol," Wireless Communications, IEEE [see also IEEE Personal Communications], vol. 13, no. 5, pp. 76-81, October 2006.

[6] D. Nguyen, T. Nguyen, and B. Bose, "Wireless broadcasting using network coding," in Third Workshop on Network Coding, Theory and Applications, 2007.

[7] D. Dujovne and T. Turletti, "Multicast in 802.11 wlans: an experimental study," in MSWiM '06: Proceedings of the 9th ACM international symposium on Modeling analysis and simulation of wireless and mobile systems, (New York, USA), pp. 130-138, ACM, 2006.

[8] J. Lacan and T. Perennou, "Evaluation of error control mechanisms for 802.11 b multicast transmissions."

[9] C. Tang and P. K. McKinley, "Modeling multicast packet losses in wireless lans," in MSWIM '03: Proceedings of the 6th ACM international workshop on Modeling analysis and simulation of wireless and mobile systems, (New York, USA), pp. 130-133, ACM, 2003.

[10] P. Fuxjäger and F. Ricciato, "Collecting broken frames: Error statistics in $802.11 \mathrm{~b} / \mathrm{g}$ links," in 4 th International Workshop on Wireless NetworkMeasurements (WiNMEE 2008) - in conjunction with WiOpt 2008, 2008.

[11] "Wireshark." www.wireshark.org. Network protocol analyzer for Windows and Unix that allows examination of data from a live network, or from a capture file on disk.
[12] T. M. Cover and J. A. Thomas, Elements of Information Theory 2nd Edition (Wiley Series in Telecommunications and Signal Processing). Wiley-Interscience, July 2006.

[13] D. Prichard and J. Theiler, "Generalized redundancies for time series analysis," Phys. D, vol. 84, no. 3-4, pp. 476-493, 1995. 